\title{
Type I collagen facilitates safe and reliable expansion of human dental pulp stem cells in xenogeneic serum-free culture
}

\author{
Mai Mochizuki ${ }^{1,2}$, Hiroshi Sagara ${ }^{3}$ and Taka Nakahara ${ }^{2 *}$
}

\begin{abstract}
Background: Human dental pulp stem cells (DPSCs) are a readily accessible and promising cell source for regenerative medicine. We recently reported that a xenogeneic serum-free culture medium (XFM) is preferable to fetal bovine serum-containing culture medium for ex vivo expansion of DPSCs; however, we observed that, upon reaching overconfluence, XFM cells developed a multilayered structure and frequently underwent apoptotic death, resulting in reduced cell yield. Therefore, we focused on optimization of the XFM culture system to avoid the undesirable death of DPSCS.
\end{abstract}

Methods: We selected type I collagen (COL) as the optimal coating substrate for the cultureware and compared DPSCs cultured on COL in XFM (COL-XFM cells) to the conventional XFM cultures (XFM cells).

Results: Our results demonstrated that COL coating facilitated significantly higher rates of cell isolation and growth; upon reaching overconfluence, cell survival and sustained proliferative potential resulted in two-fold yield compared to the XFM cells. Surprisingly, after subculturing the overconfluent COL-XFM cultures, the cells retained stem cell behavior including stable cell growth, multidifferentiation potential, stem cell phenotype, and chromosomal stability, which was achieved through HIF-1a-dependent production and uniform distribution of collagen type I and its interactions with integrins $\alpha_{2} \beta 1$ and $\alpha_{11} \beta 1$ at overconfluency. In contrast, cells undergoing apoptotic death within overconfluent XFM cultures had disorganized mitochondria with membrane depolarization.

Conclusion: The use of COL as a coating substrate promises safe and reliable handling of DPSCs in XFM culture, allowing translational stem cell medicine to achieve stable isolation, expansion, and banking of donor-derived stem cells.

Keywords: Dental pulp, Mesenchymal stem cells, Xenogeneic serum-free culture, Stem cell expansion, Collagen, Cell hypoxia, HIF-1a, Integrin, Mitochondrial dysfunction, Apoptosis

\section{Background}

Populations of dental pulp-derived stromal cells contain multipotent stem cells with a high proliferative capacity and potent differentiation potential into a wide range of cell types, such as osteo/odontogenic cells [1-4] and

\footnotetext{
* Correspondence: t.nakahara@tky.ndu.ac.jp

${ }^{2}$ Department of Developmental and Regenerative Dentistry, The Nippon

Dental University School of Life Dentistry at Tokyo, 1-9-20 Fujimi, Chiyoda-ku, Tokyo 102-8159, Japan

Full list of author information is available at the end of the article
}

non-dental cells, including neural and endodermal lineages [5-10]. The dental pulp stem cells (DPSCs, also known as dental pulp-derived mesenchymal stem cells [MSCs]) [11, 12], based on their multiple regenerative effects including paracrine signaling, immunomodulation, and immunosuppression [13-16], are considered part of the stem cell compartment and an alternative cell source of medical signaling cells $[17,18]$. Dental pulp is a readily accessible donor-tissue resource obtained by 
routine tooth extraction; therefore, DPSCs present a safe and minimally invasive MSC source for therapeutic applications and cell bank storage for future use [19-22].

It is widely known that the use of xenogeneic serum, i.e., fetal bovine serum (FBS), is necessary for MSC culture to isolate and expand donor-derived stem/stromal cells, including DPSCs [23, 24]. However, serum from an animal source includes potential risks of infection and immunological reaction associated with the cell-based therapies $[25,26]$. To avoid FBS, serum-free culture media have been developed and its use has been explored in a variety of MSC types derived from bone marrow [27-29], umbilical cord [30, 31], adipose tissue [32], periodontal ligament $[33,34]$, and dental pulp [28, 3538]. It is also recognized that cell isolation from donorderived tissues (primary culture) is difficult under serum-free conditions. Indeed, previous studies described cell isolation procedures from harvested tissues performed using FBS-containing culture media, and after the primary cultures were established, the subsequent subculture was performed in the serum-free culture media [28-30, 33, 35, 38]. Therefore, it is important to develop an efficient method for isolating MSCs from donor-derived tissues using serum-free culture conditions. Development and application of serum-free MSC culture methodology will ensure safe and reliable cellbased therapies for regenerative medicine [39, 40].

We recently demonstrated a successful isolation and expansion of donor-derived DPSCs under xenogeneic serum-free culture conditions using a commercially available culture medium [37]. Interestingly, the 14day cell growth curve analysis revealed that DPSCs reached a plateau on day 8 post-seeding and the number of cells declined on day 14. Further analysis revealed that overconfluence of cells on day 14 resulted in increased apoptosis of DPSCs, providing an explanation for the reduced cell yield. Moreover, the overconfluent DPSCs failed to proliferate after passaging. These results suggested that the serum-free cultured cells should be passaged at subconfluence and/ or confluence and a prolonged culture to overconfluence should be avoided.

The purpose of this current study was to improve the serum-free culture conditions and to create a reliable method for isolation and expansion of DPSCs. Our previous study revealed that the time required for the initial attachment of primary DPSCs cultured in xenogeneic serum-free culture medium (XFM) was significantly shorter in fibronectin-coated culture dishes compared to the non-coated ones [37]. Therefore, we decided to focus on a widely accepted method of coating culture dishes with common matrix substrates, a safe and simple way to address possible safety concerns for the future clinical applications.

\section{Methods}

\section{Cell culture}

Human dental pulp tissue was obtained from eight healthy volunteers aged 22 to 40 years undergoing routine extraction of third molars and premolars for orthodontic reasons. The isolation and expansion of DPSCs were performed as previously described [24, 37]. Briefly, the enzymatically isolated cells were cultured in XFM (PRIME-XV MSC Expansion XSFM; Irvine Scientific, Santa Ana, CA, USA) in a humidified incubator at $37{ }^{\circ} \mathrm{C}$ and $4.7 \% \mathrm{CO}_{2}$. Upon reaching $80 \%$ confluence, the cells were dissociated using $0.25 \%$ trypsin- $0.02 \%$ ethylenediaminetetraacetic acid (EDTA) buffer and then subcultured at a cell density of $5 \times 10^{3}$ cells $/ \mathrm{cm}^{2}$ in $60-\mathrm{mm}$ culture dishes coated with the substrates as determined by the following pilot experiment. All experiments were performed with cell passages P3-P5. To compensate for donor variability, all experiments were independently repeated using cells obtained from at least four individuals, with the exception of the transmission electron microscope (TEM) analysis.

\section{Initial cell growth test for determining optimal coating substrate}

In the pilot experiment, the culture dishes were coated with the following substrates: type I collagen (Cellmatrix I; Nitta Gelatin, Osaka, Japan and Corning BioCoat; Corning, NY, USA), fibronectin (PRIME-XV Human Fibronectin; Irvine Scientific and Corning BioCoat; Corning), laminin (Corning BioCoat; Corning and Bio-coat; BD Biosciences, San Jose, CA, USA), and poly-D-lysine (Corning BioCoat; Corning). For the initial cell growth test, the cells were seeded at $5 \times 10^{3}$ cells $/ \mathrm{cm}^{2}$ into respective substrate-coated culture dishes and then observed on day 4 post-seeding (D4) under the microscope. Based on the results of initial cell growth test, the culture dishes coated with the selected substrate were used in subsequent experiments. For cell adherence test, cells were seeded at $2 \times 10^{3}$ cells $/ \mathrm{cm}^{2}$ and then counted in five randomly selected fields on day 2 post-seeding (D2) using a Biorevo BZ-9000 microscope (Keyence, Osaka, Japan).

\section{Cell growth curve}

The cell growth curve was evaluated as previously described [37]. Briefly, cells were seeded at $5 \times 10^{3}$ cells/ $\mathrm{cm}^{2}$ into 12-well plates and cultured for 20 days. To generate a cell growth curve, the cells were counted in triplicate using a hemocytometer every 2 days and the results were presented as the mean \pm standard deviation (SD). 


\section{Histological examination and immunohistostaining} Cells were cultured in 60-mm dishes for 10, 14, and 20 days post-seeding (D10, D14, and D20), the time points corresponding to confluence (D10) or overconfluence (D14 and D20), and then fixed with $4 \%$ paraformaldehyde (PFA) for $10 \mathrm{~min}$ at room temperature (RT). Using fine tweezers, the fixed cultures were carefully removed from the dishes, embedded in paraffin, sectioned serially at $5-\mu \mathrm{m}$ thickness, and stained with hematoxylin and eosin (HE) as previously described [37]. Immunohistostaining was performed according to the published protocol [41] with minor modifications. Briefly, the paraffin sections were prepared as described earlier. For antigen retrieval, sections were deparaffinized, incubated at $95^{\circ} \mathrm{C}$ for $20 \mathrm{~min}$ in DAKO Target Retrieval Solution (Agilent Technologies, Santa Clara, CA, USA), and then blocked with Blocking One Histo (Nacalai Tesque, Kyoto, Japan) for $10 \mathrm{~min}$ at RT. The primary antibodies used were as follows: hypoxia-inducible factor-1 alpha (HIF-1 $\alpha)$ (1: 100; BD Biosciences), collagen type I alpha 1 (1:1000; Cell Signaling Technology, Danvers, MA, USA), collagen type IV (1:500; Abcam, Cambridge, UK), collagen type VII (1:500; Abcam), and fibronectin (1:200; Proteintech, Rosemont, USA). The secondary antibodies used were as follows: Alexa 488-conjugated donkey anti-mouse immunoglobulin (Ig) G antibody and Alexa 568-conjugated goat anti-rabbit IgG antibody (both diluted 1:1000; Life Technologies, Carlsbad, CA, USA). The stained sections were mounted with Fluoro-KEEPER Antifade Reagent containing 4',6-diamidino-2-phenylindole (DAPI) (Nacalai Tesque) and examined using a confocal laserscanning microscope (LSM 700; Carl Zeiss, Oberkochen, Germany). Sections incubated without primary antibodies were used as negative controls.

\section{Stem cell characterization}

To characterize subcultured cells for stem cell properties (stemness) after passaging of the overconfluent cultures, we assessed stem cell marker expression, multidifferentiation potential, and chromosomal stability. For marker expression analyses, the cell surface antigens and mRNA expression were evaluated by flow cytometry and reverse transcription-polymerase chain reaction (RT-PCR), respectively. For flow cytometric analysis, the following antibodies were used: CD14-FITC (clone M5E2), CD29APC (clone MAR4), CD73-FITC (clone AD2), CD90APC (clone 5E10), CD105-PE (clone 266) (all from BD Biosciences), CD34-FITC (clone 581), and CD44-FITC (clone J.173) (both from Beckman Coulter, Fullerton, CA, USA). For RT-PCR, total RNA was extracted from cell cultures and analyzed as previously described [37]. Primer sequences and PCR conditions are summarized in Table 1. Multidifferentiation induction culture into osteogenic and adipogenic lineages was evaluated as previously described [42].

Table 1 Primer sequences and amplification conditions for RT-PCR analysis

\begin{tabular}{|c|c|c|c|c|c|}
\hline \multirow{2}{*}{$\begin{array}{l}\text { Gene } \\
\text { Vimentin }\end{array}$} & \multicolumn{2}{|c|}{ Primer sequences, $5^{\prime}$ to $3^{\prime}$} & \multirow{2}{*}{$\begin{array}{l}\text { Product size (bp) } \\
200\end{array}$} & \multirow{2}{*}{$\begin{array}{l}\text { Annealing temperature }\left({ }^{\circ} \mathrm{C}\right) \\
55\end{array}$} & \multirow{2}{*}{$\begin{array}{l}\text { GenBank accession number } \\
\text { NM_003380 }\end{array}$} \\
\hline & S: & GGGACCTCTACGAGGAGGAG & & & \\
\hline & A: & CGCATTGTCAACATCCTGTC & & & \\
\hline \multirow[t]{2}{*}{ Nanog } & S: & ACCTTCCAATGTGGAGCAAC & 199 & 55 & NM_024865 \\
\hline & A: & GAATTTGGCTGGAACTGCAT & & & \\
\hline \multirow[t]{2}{*}{ Oct3/4 } & S: & GACAGGGGGAGGGGAGGAGCTAGG & 144 & 60 & NM_001173531 \\
\hline & A: & СTTCCCTCCAACCAGTTGCCCCAAAC & & & \\
\hline \multirow[t]{2}{*}{ Sox2 } & S: & AACCCCAAGATGCACAACTC & 152 & 60 & NM_003106 \\
\hline & A: & CGGGGCCGGTATTTATAATC & & & \\
\hline \multirow[t]{2}{*}{ Runx2 } & S: & CCCCACGACAACCGCACCAT & 292 & 55 & NM_001024630 \\
\hline & $A:$ & GTCCACTCCGGCCCACAAATC & & & \\
\hline \multirow[t]{2}{*}{ COL1 } & S: & CCAAATCTGTCTCCCCAGAA & 214 & 55 & NM_000088 \\
\hline & A: & TCAAAAACGAAGGGGAGATG & & & \\
\hline \multirow[t]{2}{*}{ Nestin } & S: & AACAGCGACGGAGGTCTCTA & 220 & 55 & NM_006617 \\
\hline & A: & TTCTCTTGTCCCGCAGACTT & & & \\
\hline \multirow[t]{2}{*}{ GAPDH } & S: & GTCAAGGCTGAGAACGGGAA & 613 & 55 & NM_001289746 \\
\hline & A: & GCTTCACCACCTTCTTGATG & & & \\
\hline \multirow[t]{2}{*}{$\beta$-actin } & S: & GGACTTCGAGCAAGAGATGG & 234 & 60 & NM_001101 \\
\hline & $A:$ & AGCACTGTGTTGGCGTACAG & & & \\
\hline
\end{tabular}

Oct3/4 POU class 5 homeobox 1 (POU5F1), SOX2 sex-determining region Y-box 2, Runx2 runt-related transcription factor 2, COL1 collagen type I, GAPDH glyceraldehyde-3-phosphate dehydrogenase 


\section{Karyotype analysis}

Cells grown to the mid-logarithmic stage were subjected to standard G-banding chromosome analysis at the Nihon Gene Research Laboratories (Sendai, Japan).

\section{Detection of intracellular hypoxia}

To visualize intracellular hypoxia in overconfluent cultures on D20, we used the hypoxia probe LOX-1 (SCIVAX, Kanagawa, Japan), which has its phosphorescence quenched by oxygen, as previously described [43]. Briefly, the cultures were incubated in the presence of $2 \mu \mathrm{M}$ LOX-1 for $1 \mathrm{~h}$ in a humidified incubator and then visualized by a fluorescent microscope (Keyence).

\section{Single-cell analysis of HIF-1a localization in cobalt chloride $\left(\mathrm{CoCl}_{2}\right)$-induced hypoxia}

To validate HIF- $1 \alpha$ nuclear localization, cells were incubated with $100 \mu \mathrm{M} \mathrm{CoCl}$ for $24 \mathrm{~h}$ to induce hypoxia. Immunocytostaining was performed as previously described [41]. The following primary antibodies were used: HIF-1 $\alpha$ (1:500; GeneTex, Irvine, CA, USA) and vimentin (1:10,000, Merck KGaA, Darmstadt, Germany). The following secondary antibodies were used: Alexa 568-conjugated goat anti-mouse IgG antibody and Alexa 488-conjugated goat anti-rabbit IgG antibody (both diluted 1:1000; Life Technologies). All images were acquired using the same settings on the confocal microscope (Carl Zeiss) and analyzed using ZEN 3.0 software (blue edition; Carl Zeiss). Samples incubated without a primary antibody were used as a negative control.

\section{Cell cycle analysis by flow cytometry}

The cell cycle analysis was performed using flow cytometry as previously described [24]. Flow cytometry was performed using BD FACSMelody cell sorter equipped with BD FACSChorus software (BD Biosciences). The percentage of the cells in the G0/G1, S, or G2/M phase was measured using FlowJo v10 software (Tree Star, Ashland, OR, USA).

\section{Western blotting}

Western blotting was performed as previously described [44] with minor modifications. Briefly, cell cultures were lysed in cell lysis buffer (10 mM Tris- $\mathrm{HCl}$ (pH 7.4), 1 $\mathrm{mM} \mathrm{MgCl} 2,0.1 \%$ Triton X-100) and the total amount of soluble proteins was quantified using the BSA protein assay kit (Thermo Fisher Scientific, Waltham, MA, USA). The cell lysates were separated by sodium dodecyl sulfate-polyacrylamide gel electrophoresis and transferred to a polyvinylidene fluoride membrane. Membranes were blocked with Blocking One (Nacalai Tesque) for $30 \mathrm{~min}$ at RT. The primary antibodies were collagen type I alpha 1 and $\beta$-actin (both diluted 1:1000;
Cell Signaling Technology), and Ki67 (1:2000; Abcam). The secondary antibodies were horseradish peroxidase (HRP)-conjugated goat anti-rabbit IgG antibody and HRP-conjugated horse anti-mouse IgG antibody (both diluted 1:2000; Cell Signaling Technology). The chemiluminescence was detected using AE-9300 Ez-Capture MG imaging system (ATTO, Tokyo, Japan). Density of protein bands was quantified by ImageJ software (ver. 1.52a; National Institutes of Health, Bethesda, MD, USA) and the results were normalized to $\beta$-actin. The values are presented as mean $\pm \operatorname{SD}(n=4)$.

\section{HIF-1a inhibition by YC-1 in $\mathrm{CoCl}_{2}$-induced hypoxia}

To validate the effect of YC-1, a HIF-1 $\alpha$ inhibitor (Santa Cruz Biotechnology, Santa Cruz, CA, USA), cells were cultured with $100 \mu \mathrm{M} \mathrm{YC}-1$ for $24 \mathrm{~h}$ in $\mathrm{CoCl}_{2}$-induced hypoxic conditions and HIF- $1 \alpha$ nuclear localization was assessed. Next, to investigate whether HIF- $1 \alpha$ regulated collagen type I (COL1) gene expression in our system, we treated cells cultured in $\mathrm{CoCl}_{2}$-induced hypoxic conditions (12-well plates, $3 \times 10^{5}$ cells $/ \mathrm{cm}^{2}$ ) with $100 \mu \mathrm{M}$ YC-1 for $24 \mathrm{~h}$. COL1 gene expression was assessed by RT-PCR.

\section{TEM analysis}

The overconfluent cell cultures were fixed in $100 \mathrm{mM}$ phosphate buffer solution (PB) containing 2\% PFA and $1 \%$ glutaraldehyde for $2 \mathrm{~h}$ at RT. Sections of the cell layers were dissected out, postfixed in PB containing 2\% osmium tetroxide, dehydrated in graded series of ethanol, and embedded in Epon 812 (TAAB, England, UK) resin mixture. Ultrathin sections were cut by an ultramicrotome, stained with uranyl acetate and lead citrate. The sections were examined using transmission electron microscope (Hitachi H-7500, Tokyo, Japan).

\section{Mitochondrial membrane potential}

The mitochondrial membrane potential $(\Delta \Psi \mathrm{m})$ was assessed by flow cytometry using the JC-1 MitoScreen assay (BD Biosciences) according to the manufacturer's instructions. Flow cytometry on JC-1-stained cells was performed using BD FACSMelody cell sorter equipped with BD FACSChorus software (BD Biosciences). Single-cell samples were gated to exclude cell debris and aggregates using FlowJo v10 software (Tree Star). The quantification of the JC-1 monomers fold change from D10 to D20 in each cell type was determined using the following formula: the ratio of JC-1 monomers to JC-1 aggregates on D20 was divided by the corresponding ratio on D10. The values were expressed as mean $\pm \operatorname{SD}(n=4)$. 
Terminal deoxynucleotidyl transferase deoxyuridine triphosphate nick end labeling (TUNEL) staining The deparaffinized sections of cell cultures fixed on D10, D14, and D20 were prepared as described earlier. TUNEL staining was performed according to the manufacturer's instructions (Roche Diagnostics, Mannheim, Germany). The stained sections were examined using a confocal microscope (Carl Zeiss).

\section{Statistical analysis}

Statistical analyses were performed using IBM SPSS Statistics software (version 23.0; IBM Japan, Tokyo, Japan). For a two-group comparison, an unpaired $t$ test was applied. Mann-Whitney $U$ test was used for nonparametric data. For all statistical analyses, $p<0.05$ indicated statistical significance.

\section{Results}

Type I collagen facilitates initial cell growth under XFM culture conditions

To investigate the benefits of coating cultureware to improve XFM culture conditions, first, we tested several commonly used substrates, including type I collagen (COL), fibronectin, laminin, and poly-D-lysine. After a 4-day incubation period, DPSCs grew in culture plates coated with $\mathrm{COL}$ and fibronectin, while the cells did not adhere in the plates coated with laminin and poly-D-lysine (Additional file 1: Figure S1). In the present study, DPSCs cultured on COL in XFM were designated as "COL-XFM cells", while
DPSCs cultured on fibronectin in XFM were designated as conventional "XFM cells" [37].

To further characterize the substrates, the cells isolated from the dental pulp tissue by enzymatic digestion were seeded on COL or fibronectin and the adherent cells were evaluated as previously described [37]. We observed that the time period required to show cell division of adherent cells in COL-XFM primary culture was significantly shorter compared to the XFM primary culture $(4.0 \pm 1.0$ vs $7.8 \pm 1.3$ days; $n=4 ; P=0.0073)$. Furthermore, after seeding cells on COL at low cell density, COL-XFM cells had a significantly higher number of adhered cells compared to the XFM cells after 2 days of culture $(p<0.01$; Additional file 2: Figure S2). In summary, COL provided consistent cell isolation and growth at the initial cell proliferation phase in XFM culture conditions. Therefore, we selected COL as the optimal coating substrate for our subsequent experiments.

\section{COL facilitates robust cell growth and prolonged cell expansion}

To characterize the growth curves of COL-XFM and XFM cells, we cultured the cells for up to D20. XFM cells showed active growth and reached a plateau during 12 days of culture; the number of cells decreased on D14 as previously reported [37] and remained constant until D20 (Fig. 1a). Interestingly, COL-XFM cells exhibited robust and sustained growth and reached a plateau on day 16 without any reduction of cell number throughout 20 days of culture, yielding double the number of cells

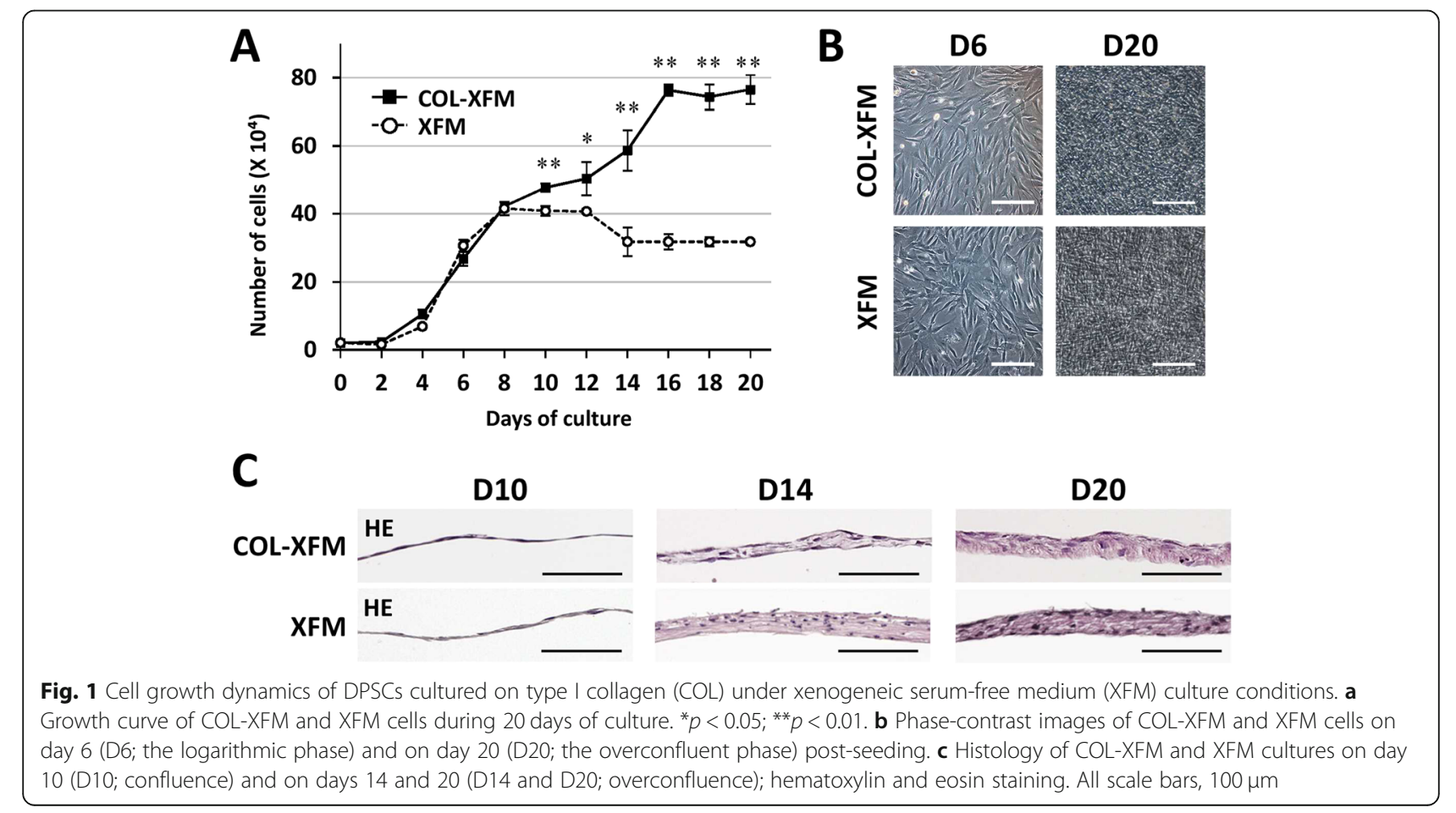


compared to the XFM cells (Fig. 1a). Phase-contrast observation on D6 and D20 and the histological examination on D10 showed no obvious differences in both cultures (Fig. 1b, c). Both cell types developed a multilayered structure by D14 and produced abundant extracellular matrix (ECM) by D20, as demonstrated by the eosin staining (Fig. 1c). Furthermore, we observed the condensed nuclei in the XFM cells on D14 and D20, but not in the COL-XFM cells. At the same time, the COLXFM multilayered structure became thicker between D14 and D20, while the XFM tissue thickness did not appear to change during that time period (Fig. 1c). In summary, we found that under XFM culture conditions, COL facilitates robust cell growth of DPSCs even after overconfluence and the development of well-organized multilayered ECM.

\section{The multilayered COL-XFM cells maintain stable growth and stem cell properties after subculture}

In our previous study, we reported that, upon passaging the overconfluent XFM cultures on D14, XFM cells formed aggregates consisting of senescent or dead cells, and lost proliferative capacity [37]. To investigate the effect of COL coating on cell growth and stem cell properties, both XFM multilayers were subcultured on D20. Surprisingly, COL-XFM cells were passaged normally and showed stable subsequent proliferation (Fig. 2a). In contrast, XFM cells formed aggregates and did not proliferate any further (Fig. 2a), consistent with our previous study [37]. Furthermore, the subcultured COL-XFM cells showed normal karyotype with diploidy (Fig. 2b), presence of common stem cell marker antigens (Fig. 2c), typical gene expression profile (Fig. 2d), and multidifferentiation potential (Fig. 2e), all of the parameters consistent with the DPSC properties [24, 37]. In summary, these results indicated that after subculture, the multilayered COL-XFM cells maintained stem cell properties (stemness), including the proliferative ability, multidifferentiation potential, and stem cell phenotype along with chromosomal stability.

\section{COL coating induces nuclear localization of HIF-1 $a$ and proliferative potential in COL-XFM cells}

To investigate the mechanisms regulating cells at overconfluence, first, we assessed the hypoxic conditions in D20 multilayered cultures using LOX-1 staining. As expected, LOX-1 phosphorescence, an indication of a hypoxic state, was detected in both XFM and COL-XFM multilayers (Fig. 3a). Next, we hypothesized that HIF-1 $\alpha$, a master hypoxia regulator, plays an important role in the cellular responses in COL-XFM and XFM multilayers. Immunohistostaining revealed that HIF- $1 \alpha$ was localized predominantly in the nuclei of the COL-XFM multilayer, whereas in the XFM multilayer, HIF- $1 \alpha$ was also detected in the cytoplasm (Fig. 3a). To confirm nuclear localization of HIF- $1 \alpha$, we performed a single-cell analysis of COL-XFM and XFM cells. Cells were seeded at a low cell density and treated with $\mathrm{CoCl}_{2}$ to mimic the hypoxic conditions. As expected, HIF- $1 \alpha$ was localized to the nucleus of the COL-XFM cell, and the fluorescence intensity of HIF- $1 \alpha$ overlapped with DAPI staining (Fig. 3b). By contrast, in the XFM cell, HIF- $1 \alpha$ was also detected in the cytoplasm and not exclusively in the nucleus (Fig. 3b). In summary, our results demonstrated that nuclear localization of HIF- $1 \alpha$ can be induced by hypoxic conditions in COL-XFM cultures, suggesting that HIF- $1 \alpha$ could be involved in cellular responses at overconfluency.

It is well known that HIF- $1 \alpha$ is a potent proliferative regulator under hypoxia $[45,46]$. Therefore, we decided to evaluate the cell cycle status and time-course expression of Ki67, a proliferation marker tightly linked to the cell cycle, in COL-XFM and XFM cells on D10 (confluence), D14, and D20 (overconfluence). First, we performed a cell cycle analysis using flow cytometry. A considerable percentage of COL-XFM cells was in the G2/M and S phases on D10, D14, and D20. In contrast, the percentage of XFM cells in the G2/M and S phases was markedly decreased on D14 and thereafter, resulting in the cell cycle arrest in the G0/G1 phase (Fig. 3c). This was further verified by western blotting. COL-XFM cells showed stable expression of Ki67 on D10 and D14, with low expression levels still detectable on D20. At the same time, in the XFM cells, the Ki67 expression was observed on D10, but it was dramatically downregulated on D14 and was almost undetectable on D20 (Fig. 3d). These results suggested that the robust cell growth after reaching confluence in COL-XFM cells was regulated by active cell cycle status and sustained expression of Ki67; however, the cell cycle arrest and lack of Ki67 expression resulted in decreased growth of the XFM cells on D14 and thereafter.

\section{Integrins $\alpha 2 \beta 1$ - and $\alpha 11 \beta 1$-mediated interactions with COL1, regulated by HIF-1 $\alpha$, are responsible for cell survival in COL-XFM cells during prolonged culture} To investigate whether the ECM composition was different between the two XFM cultures, we focused on COL1, the major component of ECM. Immunohistostaining revealed that on D10 there was no apparent difference in COL1 staining between the two XFM confluent monolayers (Fig. 4a). However, by D20, COL1 staining was stronger and more uniform throughout the COL-XFM multilayer, while the immunoreactivity in the XFM multilayer was limited to the apical (upper) and basal (attached) surfaces and, surprisingly, was completely absent in the intermediate layer (Fig. 4a). Next, we assessed COL1 expression by immunoblotting 


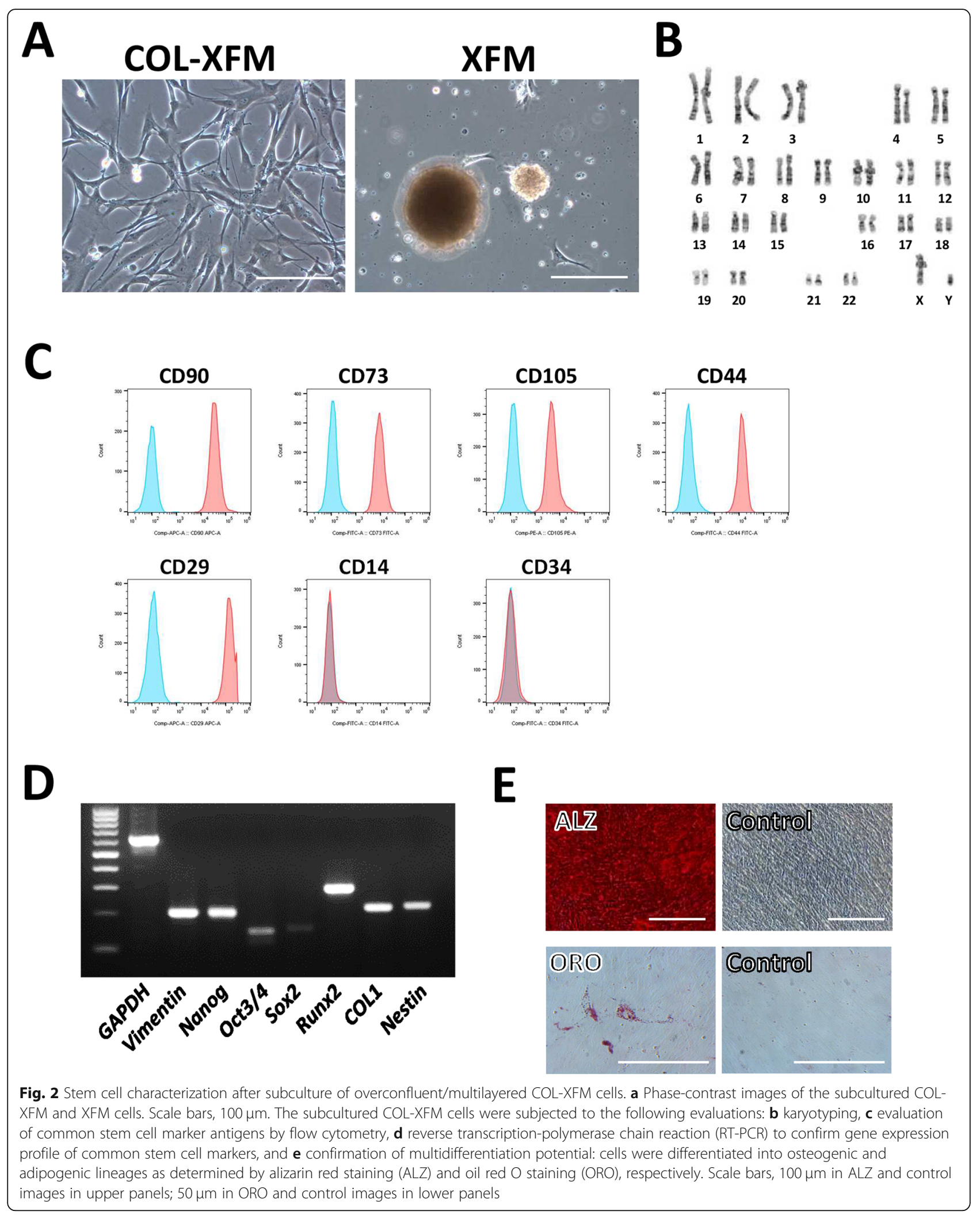




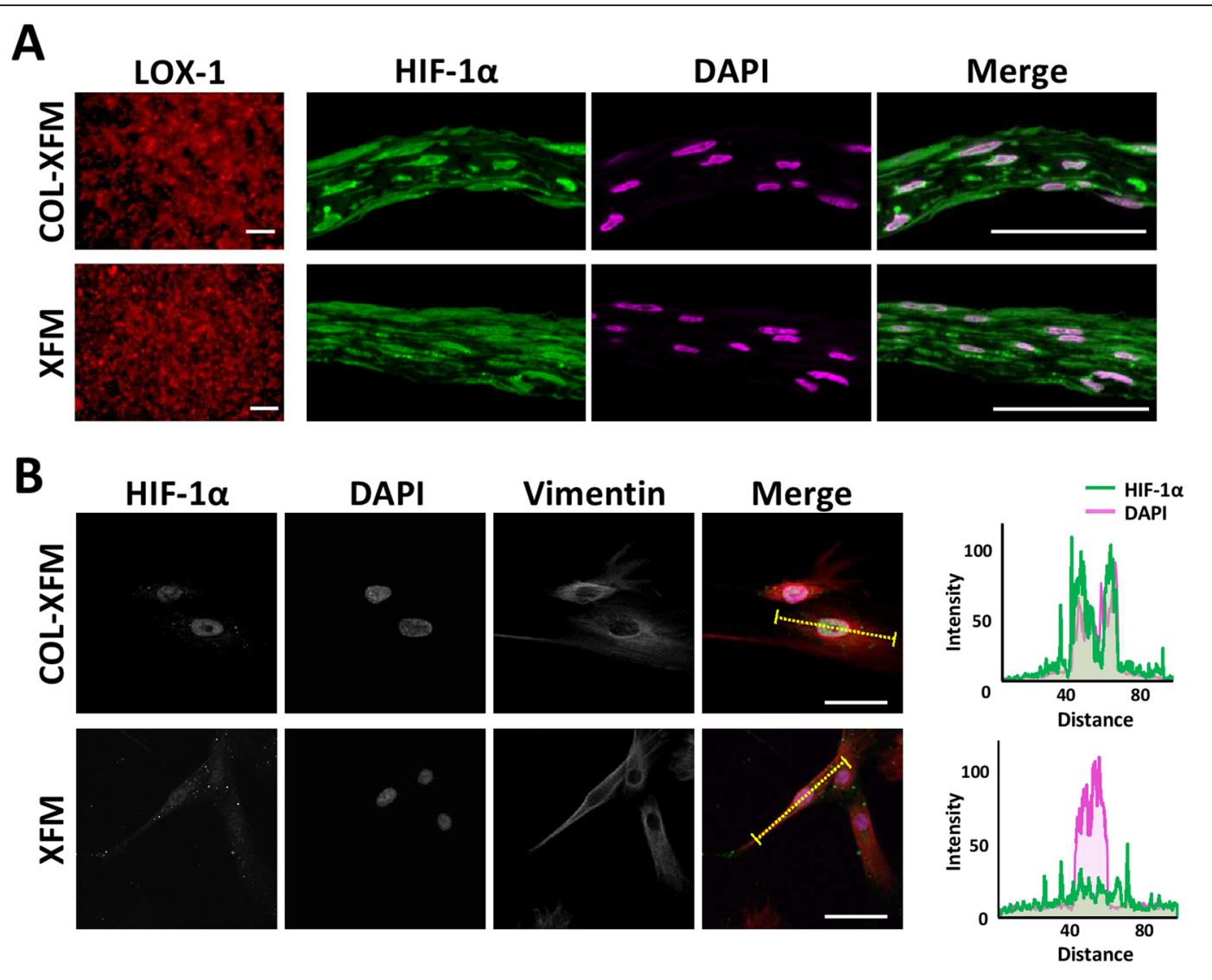

C
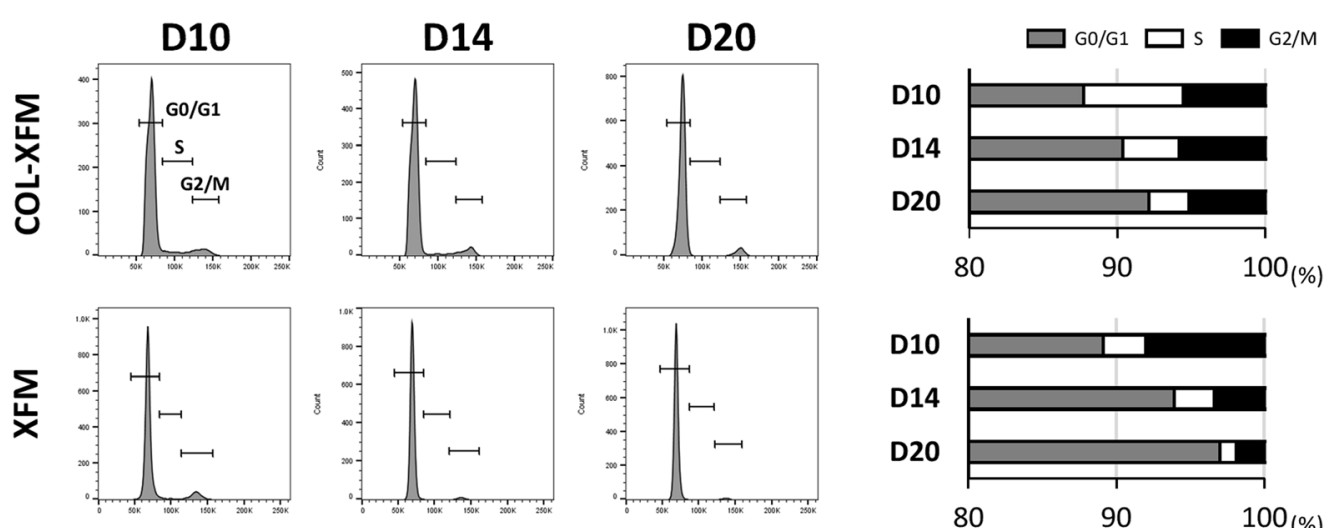

D
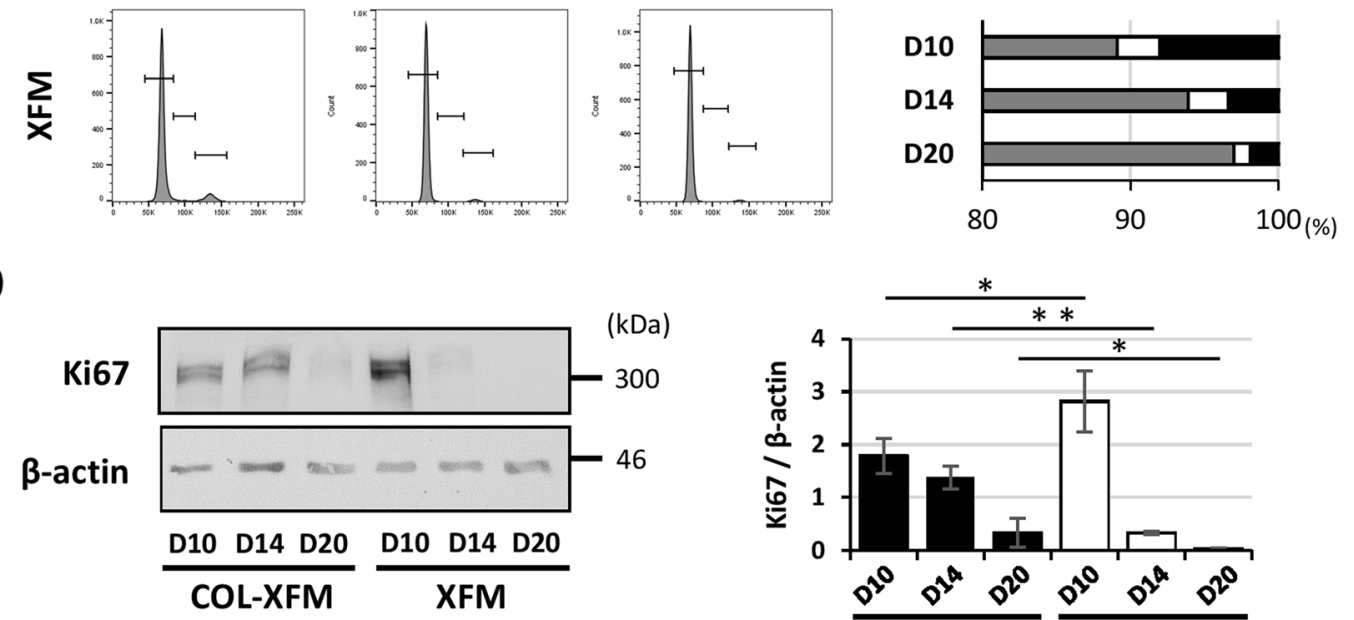

Fig. 3 (See legend on next page.)

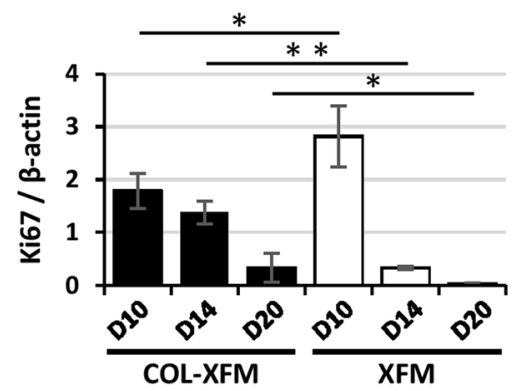




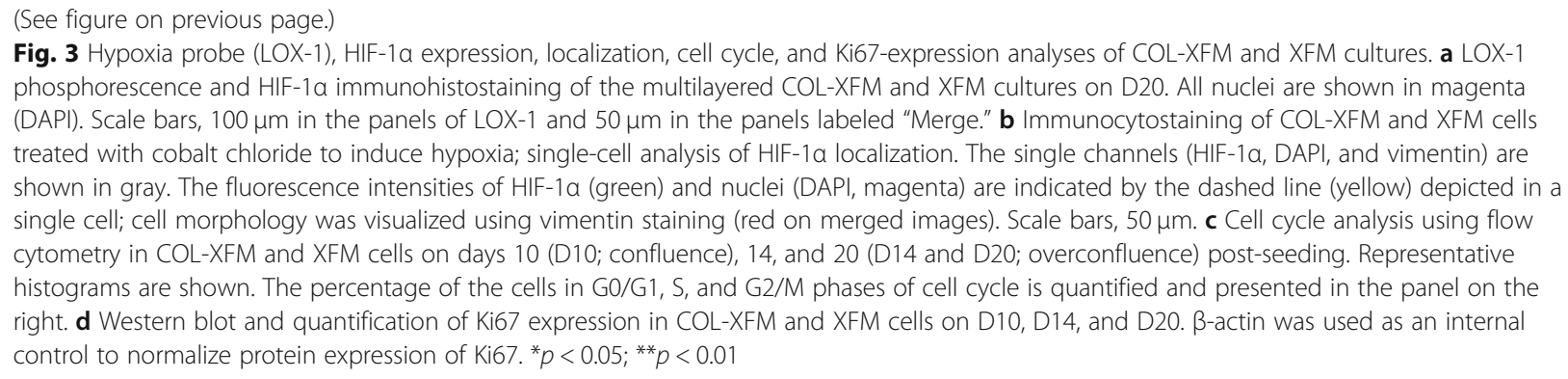

(Fig. 4b). Our results demonstrated that COL1 levels were significantly increased in COL-XFM cells on D20 compared to D10 $(p<0.01)$, while in the XFM cells, there was no significant difference in COL1 protein levels between D10 and D20.

Next, to elucidate the role of HIF-1 $\alpha$ in COL1 production in the XFM cells, we investigated COL1 mRNA expression in the presence of commonly used HIF- $1 \alpha$ inhibitor YC-1 by RT-PCR. First, we tested the effect of YC- 1 on HIF-1 $\alpha$ nuclear translocation in cells cultured under $\mathrm{CoCl}_{2}$-induced hypoxia conditions. As expected, YC-1 completely blocked the nuclear localization of HIF- $1 \alpha$ in a COL-XFM cell (Additional file 3: Figure S3). Furthermore, in the presence of YC-1, the expression of COL1 was markedly decreased in both types of XFM cells (Fig. 4c), suggesting that the production of COL1 was regulated by HIF-1 $\alpha$. We also assessed D20 expression of collagen types IV, VII, and fibronectin, the components of ECM involved in its function and stability. Interestingly, there were no apparent differences in the distribution patterns of these ECM proteins in both multilayers (Additional file 4: Figure S4).

Considering the uneven distribution of COL1 in the XFM multilayer, we hypothesized that the lack of interactions between the cells and the ECM had resulted in the apoptotic death of the cells, a phenomenon known as anoikis. It was reported that integrins $\alpha 2 \beta 1$ and $\alpha 11 \beta 1$, the specific receptors to COL1, were required for cell death/survival in bone marrow-derived stromal cells (BMSCs) [47]. Therefore, we investigated whether the expression of $\alpha 2$ and $\alpha 11$ integrin subunits was affected in both XFM multilayers. Western blot analysis clearly revealed that the expression of $\alpha 2$ and $\alpha 11$ integrin subunits on D20 was significantly lower in the XFM cells compared to the COL-XFM cells (Fig. 4d). These results suggest that COL1 binding interactions mediated by integrins $\alpha 2 \beta 1$ and $\alpha 11 \beta 1$ might be responsible for cell survival in COL-XFM multilayer.

\section{Disorganized mitochondria with membrane depolarization contributed to cell death in overconfluent XFM but not in COL-XFM cultures}

To explore the cause of cell death in the XFM cultures, we investigated cellular ultrastructure of multilayers on
D20 by TEM. As shown in Fig. 5a, both cultures exhibited proper stratified appearance consisting of squamous cells. COL-XFM cells showed normal structure of organelles including the mitochondria, whereas the cytoplasm of the XFM cells had a considerable number of dense structures, containing disorganized mitochondria of different shapes and sizes. These results suggested that the cell death in the XFM multilayer could be caused by the mitochondrial abnormality.

Next, we decided to further investigate cell morphology and mitochondrial function. Both cultures were trypsinized on D20 and cell suspensions were analyzed by flow cytometry to determine the distribution profiles of cell size and internal complexity/granularity based on the forward scatter (FSC-A) and side scatter (SSC-A) dot plots, respectively. The results indicated that COLXFM cells showed a normal distribution of population with a high homogeneity in both the cell size (FSC-A) and internal granularity (SSC-A) (Additional file 5: Figure S5). In contrast, the majority of the XFM cells were smaller compared to the COL-XFM cells and showed a higher degree of heterogeneity based on the FSC-A dot plot. Furthermore, based on the SSC-A profile data, XFM cells had a heterogeneous distribution of cell internal structures (Additional file 5: Figure S5), possibly due to high cytoplasmic granularity as a result of numerous disorganized mitochondria detected by the TEM (Fig. 5a).

To assess mitochondrial health, we used JC-1 fluorescent dye to analyze mitochondrial membrane potential by flow cytometry. First, the appropriate gates were set to include cells while excluding cell debris and aggregates (Additional file 6: Figure S6A). COL-XFM cells cultured for 1 week without media change were used as a positive control to verify depolarized (unhealthy) mitochondria, while unstained cells served as a negative control (Additional file 6: Figure S6B). Our results demonstrated that there were no differences in distribution of JC-1 aggregates in COL-XFM cells at both D10 (confluence) and D20 (overconfluence) time points, whereas the distribution of JC-1 aggregates dramatically decreased in XFM cells between D10 and D20 (Fig. 5b). We quantified the ratio of the JC-1 monomers on D20 

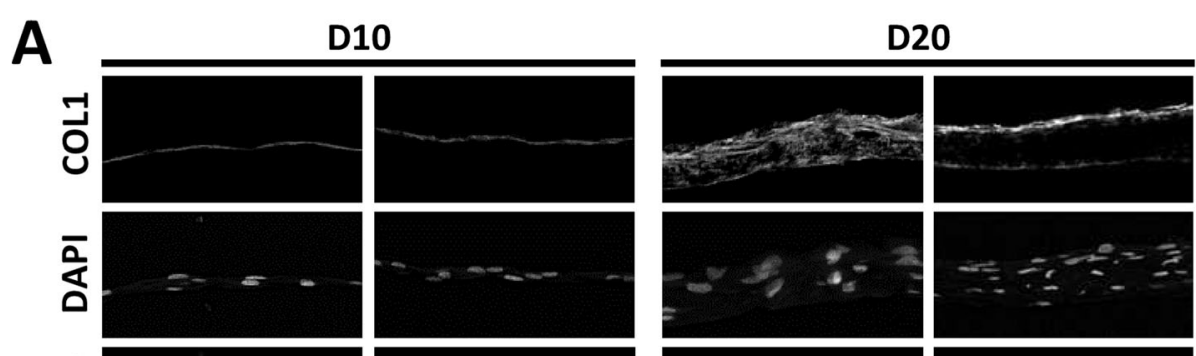

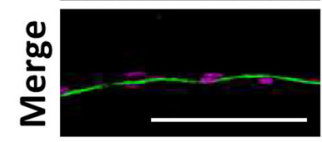

COL-XFM

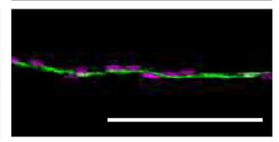

XFM

B

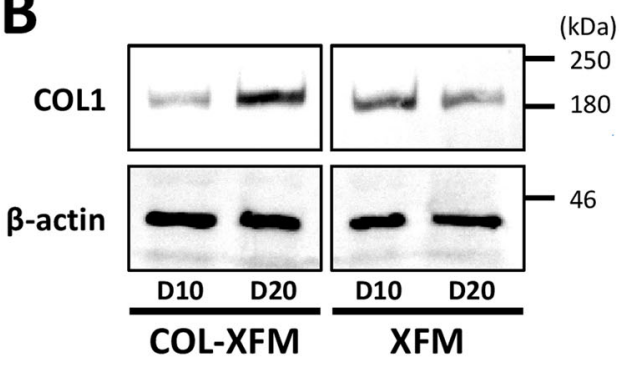

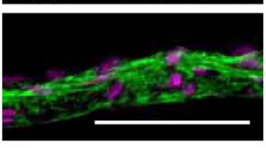

COL-XFM

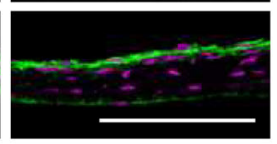

XFM
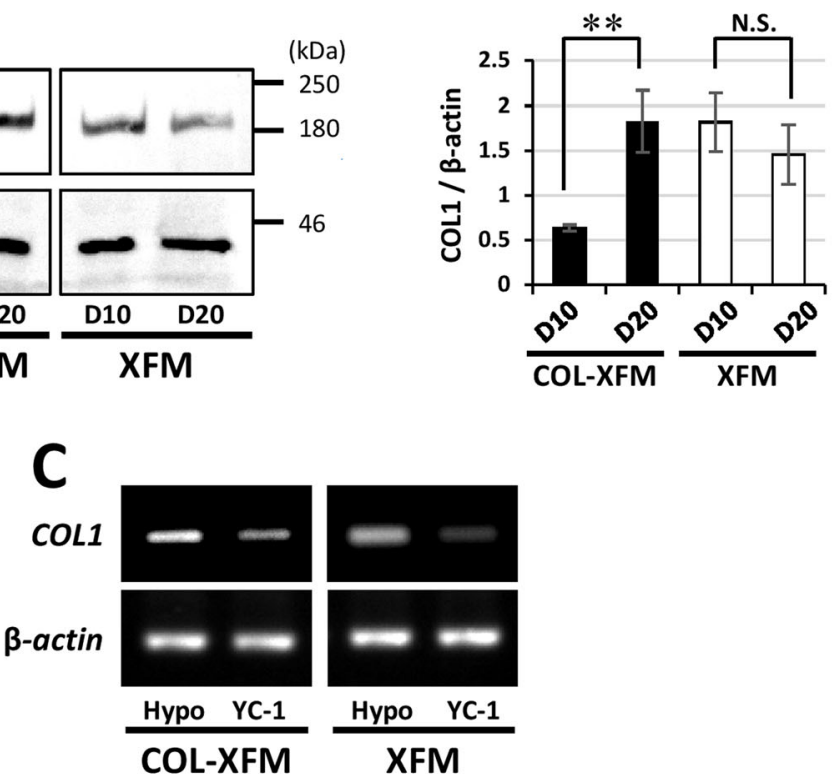

D
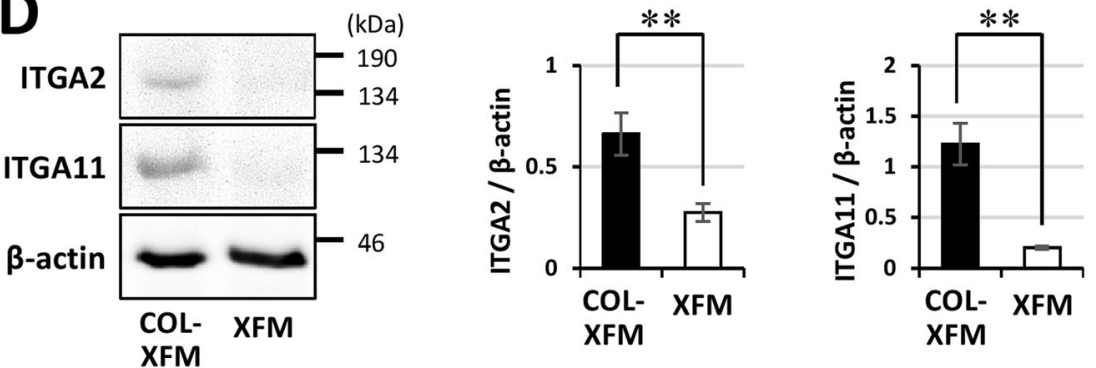

Fig. 4 Collagen type I (COL1) expression, regulation, and interactions in COL-XFM and XFM cultures on D10 and D20. a Immunohistostaining of both confluent/monolayered and overconfluent/multilayered XFM cultures on D10 and D20, respectively. The single channels (COL1 and DAPI) are shown in gray; all nuclei in the merged images are shown in magenta (DAPI); scale bars, $100 \mu \mathrm{m}$. b Immunoblotting and quantification of COL1 protein expression in the cultures described in $\mathbf{a}$. $\beta$-actin was used as a loading control to normalize protein expression of COL ${ }^{* * *} p<0.01$; N.S., no significant difference. c RT-PCR was used to measure gene expression of COL1 in COL-XFM and XFM cells in the absence (Hypo) or presence (YC-1) of a HIF-1a inhibitor YC-1 during cobalt chloride-induced hypoxia. $\mathbf{d}$ Western blot and quantification of integrin a2 and a11 subunits (ITGA2 and ITGA11) in both types of multilayered XFM cultures on D20. $\beta$-actin was used as a loading control to normalize the expression of ITGA2 and ITGA11. ** $p<0.01$

to those on D10 and demonstrated that the ratio was significantly higher in XFM cells compared to the COLXFM samples $(p<0.05$; Fig. 5 c). These results indicated that mitochondrial depolarization in XFM cells was significantly increased between D10 (confluence) and D20 (overconfluence), while COL-XFM cells maintained 

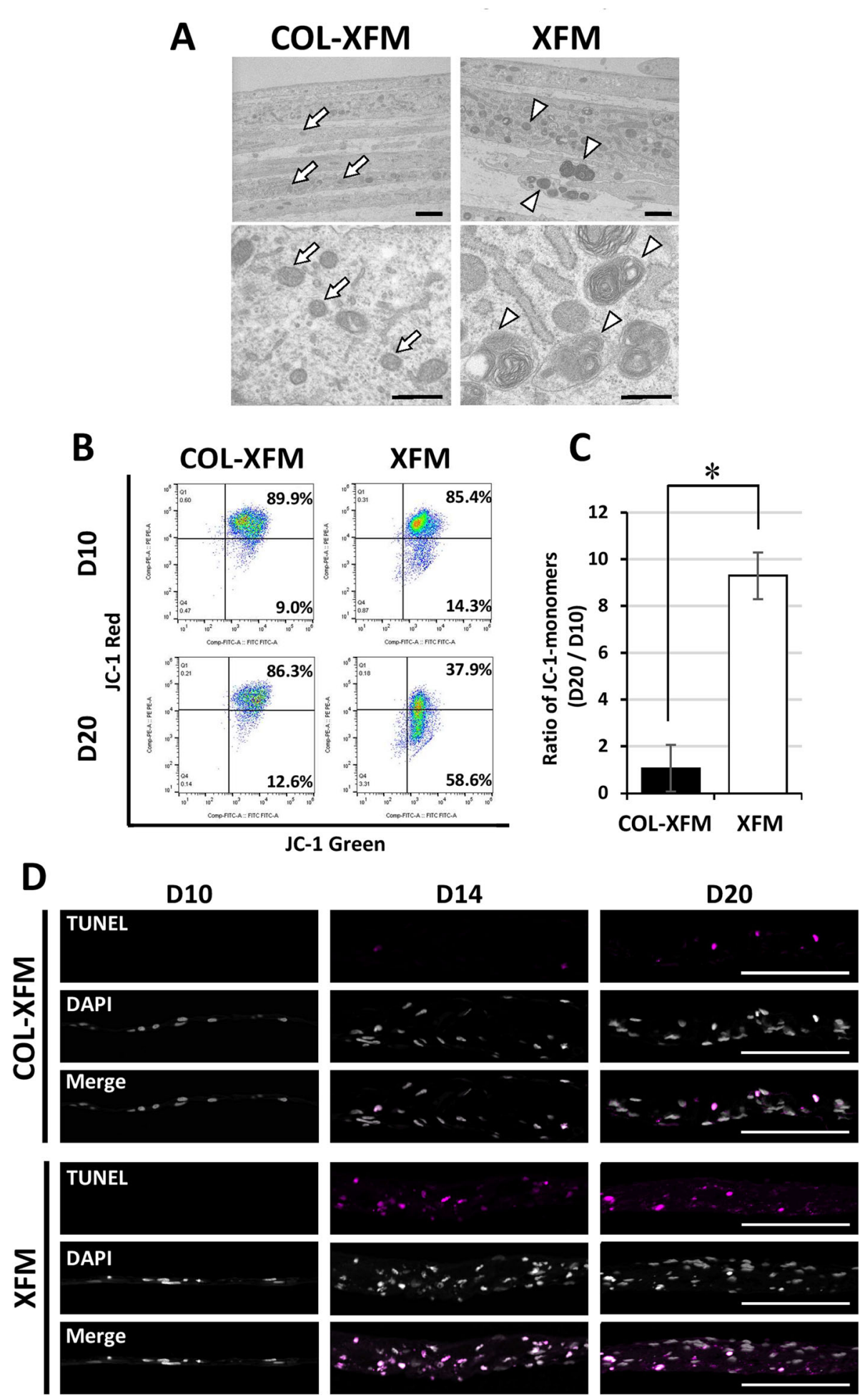

Fig. 5 Ultrastructure, cell morphology, and mitochondrial function of DPSCS cultured on COL coating under the XFM culture conditions. a TEM of both types of multilayered XFM cultures on D20 post-seeding. Arrows indicate healthy mitochondria; arrowheads indicate disorganized mitochondria. Scale bars, $1.7 \mu \mathrm{m}$ for the upper panels and $500 \mathrm{~nm}$ for the lower panels. b JC-1 stained cells were analyzed by flow cytometry to evaluate mitochondrial membrane potential in COL-XFM and XFM cultures on days 10 (D10) and 20 (D20). c Quantification of the JC-1 monomers ratio, presented as fold difference of D20 vs. D10 in each cell culture condition. ${ }^{*} p<0.05$. $\mathbf{d}$ Terminal deoxynucleotidyl transferase-mediated deoxyuridine triphosphate nick end labeling (TUNEL) staining of COL-XFM and XFM cultures on D10, D14, and D20. TUNEL-positive nuclei are shown in magenta. All nuclei are shown in gray (DAPI). Scale bars, $100 \mu \mathrm{m}$ 
healthy mitochondria with stable membrane potential even after confluence. Based on these results, we decided to examine the level of apoptosis in both cultures. As expected, multilayered XFM cells on D14 and D20 appeared to have a higher number of apoptotic cells, as evaluated by TUNEL staining, compared to the COLXFM multilayer (Fig. 5d). In summary, disorganized mitochondria with membrane depolarization contributed to cell death in overconfluent XFM cultures; however, the presence of COL prevented such changes in COLXFM cells.

\section{Discussion}

Here, in this study, we present evidence that COLXFM culture promoted initial attachment and subsequent growth without a reduction in cell yield during a prolonged 20-day culture; however, the conventional XFM culture showed growth arrest upon reaching a plateau and a declined cell yield due to apoptotic cell death and mitochondrial dysfunction. Regarding the mechanisms regulating the distinct outcomes, we demonstrated that HIF- $1 \alpha$ played an important role during overconfluence, a hypoxic microenvironment, as shown by HIF-1 $\alpha$-nuclear localization in COLXFM cells. The assay using the HIF- $1 \alpha$ inhibitor YC1 further confirmed that the expression of COL1 was regulated by HIF- $1 \alpha$. As a result, HIF- $1 \alpha$ significantly upregulated COL1 production in the COL-XFM multilayer, but not in the XFM multilayer, as shown by immunostaining and immunoblotting (Fig. 4). At the same time, expression and spatial distribution of other ECM proteins, including collagen types IV, VII, and fibronectin, were not affected in both types of XFM multilayers (Additional file 4: Figure S4). These results suggested that HIF-1 $\alpha$-dependent COL1 production and its distribution within the multilayered COL-XFM structure were essential for cell survival and proliferative potential in the hypoxic environment. Our results also demonstrated that cell fate and function were closely associated with the collagen-integrin interactions, as discussed below.

In general, upon establishing cell adhesion to ECM, the integrins activate several intracellular signaling pathways involved in the regulation of cell proliferation, migration, differentiation, as well as survival and death [48-50]. In particular, cell adhesion to COL1 is mediated by integrins $\alpha 1 \beta 1, \alpha 2 \beta 1$, and $\alpha 11 \beta 1$ [51]. Previous study reported that in BMSCs, interference with COL1 binding to the integrins $\alpha 2 \beta 1$ and $\alpha 11 \beta 1$ resulted in cell death (anoikis) due to mitochondrial depolarization (this study was performed in the presence of FBS) $[47,52]$. In line with these findings, we observed that multilayered XFM cultures showed significantly lower expression of $\alpha 2$ and $\alpha 11$ integrin subunits compared to the multilayered COL-XFM cultures
(Fig. 4d). Our findings also showed that the lack of $\alpha 2 \beta 1 /$ $\alpha 11 \beta 1$-mediated interactions with COL1 resulted in cell death accompanied by disorganized mitochondria with membrane depolarization (Fig. 5). As demonstrated in the overconfluent/multilayered COL-XFM cultures, COL1rich microenvironment likely provided the stem cell niche within the multilayered structure. Therefore, COL1-integrins $\alpha 2 \beta 1 / \alpha 11 \beta 1$ interactions were essential for not only cell survival, but also for stemness maintenance in COLXFM cells. In contrast, XFM cells were unable to establish appropriate $\alpha 2 \beta 1 / \alpha 11 \beta 1$-mediated interactions, because of the lack of COL1, resulting in anoikis. Given that collagen production, cell survival, and self-renewal ability in hypoxia were regulated by HIF- $1 \alpha[46,53,54]$, overconfluent COL-XFM cells avoided apoptotic death and strengthened the proliferative potential as verified by active cell cycle status and sustained Ki67-expression.

In Fig. 6, we summarized the results of our study and proposed the following mechanism. During the XFM culture conditions, COL coating promotes the initial attachment, stable growth, and survival of DPSCs. In a hypoxic environment (overconfluence), cell survival and sustained proliferative potential are achieved through HIF-1 $\alpha$-dependent production of COL1 via integrins $\alpha 2 \beta 1 / \alpha 11 \beta 1$ interactions, allowing cells to retain stemness and chromosomal stability. Without COL coating, XFM cells display mitochondrial abnormality and undergo apoptosis.

The conventional XFM culture is a highly productive method for isolation and expansion of donor-derived DPSCs during the monolayer culture and passaging at subconfluency [37]. On the other hand, here, we show that applying COL coating method to the XFM culture ensures safer and predictable handling of DPSCs and has three main advantage points. First, the initial cell adhesion and subsequent growth are promoted after isolating and passaging the COL-XFM cells. Second, the COLXFM cells in overconfluent/multilayered state can be protected from apoptotic death, avoiding reductions of cell yield. Third, overconfluent COL-XFM cells can be subcultured and further expanded while maintaining stemness. Therefore, the COL coating method is effective for establishing safe and reliable cell culture protocols using XFM, based on the following points: (1) it allows consistent and predictable donor-derived cell isolation and passaging in XFM culture; (2) it allows longer culture periods, resulting in overconfluence and cell yields approximately two-fold higher at a single timepoint (i.e., without passaging); and (3) it allows reduction of cell-passage frequency, avoiding cell damage due to trypsin-EDTA exposure. Furthermore, this protocol avoids the exposure of DPSCs to the xenogeneic serum (i.e., FBS) throughout the whole culture period, including donor-tissue collection (transport to the laboratory), 


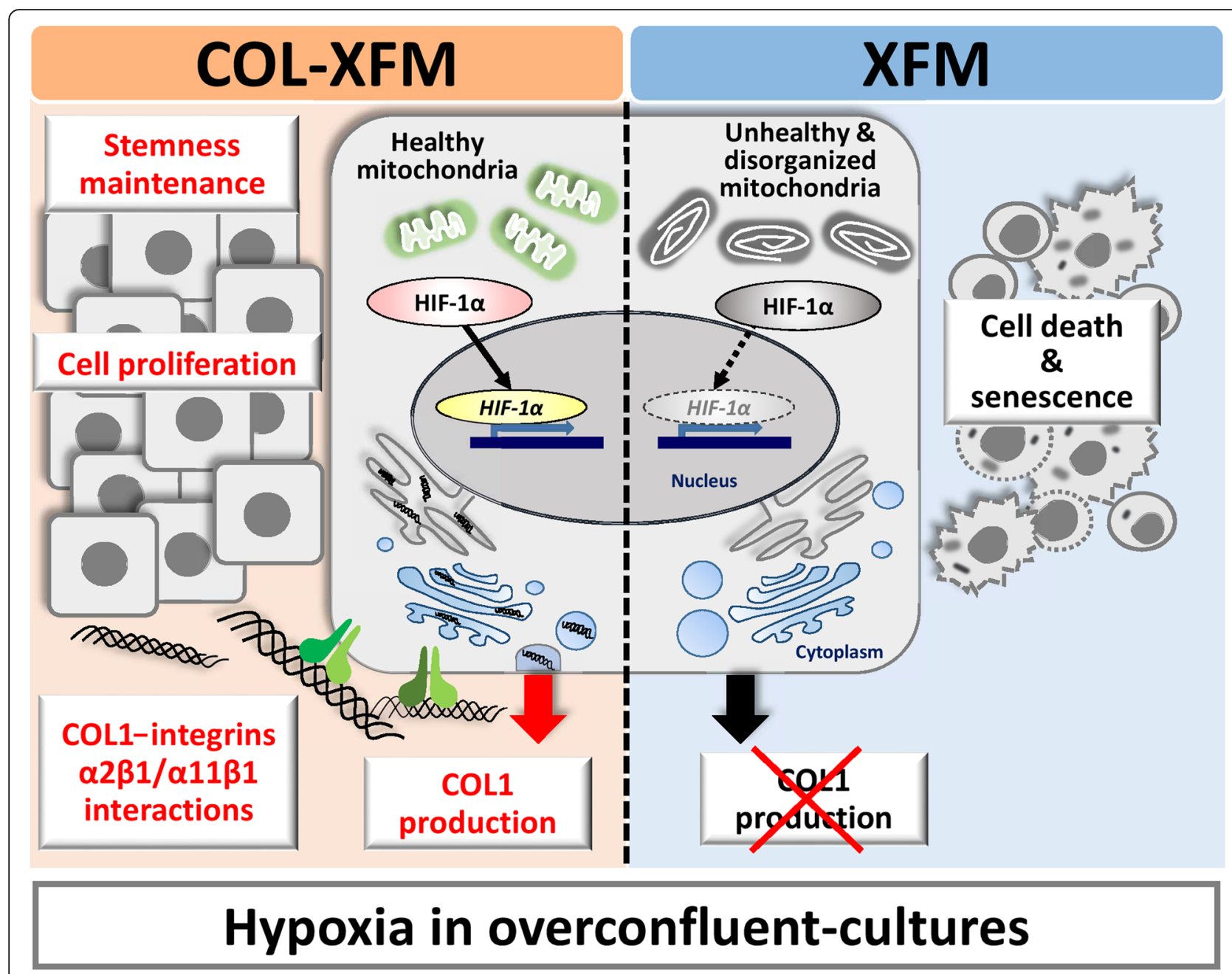

Fig. 6 Schematic diagram summarizing the role of ECM, hypoxia, HIF-1a-dependent production of collagen type I (COL1), and integrins a2 $\beta 1$ and a1 $1 \beta 1$ in cell survival, proliferative potential, and stemness maintenance in DPSCs. In the absence of COL coating, upon reaching overconfluence, the multilayer formation generates the hypoxic environment, leading to apoptotic death accompanied by mitochondrial abnormality in the XFM cells. COL coating promotes cellular attachment, proliferation, and survival of COL-XFM cells. Furthermore, nuclear localization of HIF-1a within the COL-XFM multilayer activates the production of COL1. The resulting COL1-integrins a2 $\beta 1 / \alpha 11 \beta 1$ interactions lead to cell survival and increased proliferative potential. Upon passaging, the COL-XFM cells retain stemness (i.e., proliferative ability, multidifferentiation potential, and stem cell phenotype) and chromosomal stability. Without COL coating, XFM cells display apoptosis and mitochondrial abnormality

cell isolation (primary culture), expansion (subculture), and storage (cryopreservation) [37]. Hence, our current research strategy is fully committed to translational donor-derived/tissue-specific stem cell medicine based on cell isolation, expansion, and banking for future use.

This study of COL-XFM multilayered cultures, a COL1-rich stem cell niche, demonstrated the existence of the HIF-1 $\alpha$-dependent ECM production pathway and confirmed the role of ECM-integrin interactions in cell survival, retention of stemness, and chromosomal stability. In contrast, in the conventional XFM multilayers, the absence of COL1 resulted in cell death (anoikis), accompanied by mitochondrial abnormality. DPSCs are only one example of typical donor-derived MSC sources; therefore, these current findings are highly applicable to XFM culture of other MSCs, especially BMSCs $[47,52]$. A recent study reported that collagen plays an important role in BMSCs proliferation, survival, and adhesion, compared to other coating substrates, such as fibronectin and poly-L-lysine [55]. Furthermore, other studies demonstrated that native ECM produced by the BMSCs, the matrix consisting primarily of collagen, efficiently supported stem cell properties, such as clonogenicity, cell growth, and osteogenic differentiation potential $[56,57]$. Therefore, further investigation of regulatory pathways involved in DPSCs survival and stemness maintenance provides methodological and practical insights into tissue-specific stem cell biology and donor-derived stem cell medicine, contributing to 
the development of safe and predictable MSC-based therapies in regenerative medicine.

\section{Conclusion}

COL coating of the cultureware enables us to achieve consistent cell isolation and robust expansion of donorderived DPSCs, even after reaching confluence, leading to increased yield of XFM-cultured cells. In the COL-XFM cultures at overconfluency, HIF- $1 \alpha$-dependent expression of COL1 and integrin-mediated anchorage to COL1 are involved in cell survival and sustained proliferative potential, contributing to stem cell properties, as well as chromosomal stability after subculture. This readily and widely applicable method promises a safe and reliable XFM culture in order to establish appropriate procedures for isolating, expanding, and banking donor-derived cell source for stem cell research and therapy.

\section{Supplementary information}

Supplementary information accompanies this paper at https://doi.org/10. 1186/s13287-020-01776-7.

Additional file 1: Figure S1. Phase-contrast images of DPSCs cultured in xenogeneic serum-free culture conditions on the coated substrates as follows: type I collagen supplied by (a) Nitta Gelatin and (b) Corning; fibronectin supplied by (a) Irvine Scientific and (b) Corning; laminin supplied by (a) Corning and (b) BD Biosciences and poly-D-lysine supplied by Corning. In the initial cell growth test, cells were seeded at $5 \times 10^{3}$ cells $/ \mathrm{cm}^{2}$ (conventional cell density when passaging) and the images were taken on day 4 post-seeding. Scale bars, $200 \mu \mathrm{m}$.

Additional file 2: Figure S2. Phase-contrast images and quantification of DPSCs cultured on type I collagen and fibronectin in xenogeneic serum-free culture conditions. In cell adherent test, cells were seeded at $2 \times 10^{3} \mathrm{cells} / \mathrm{cm}^{2}$ (a sparse cell density) and the images were taken on day 2 post-seeding. Scale bars, $200 \mu \mathrm{m}$. ${ }^{* *} p<0.01$.

Additional fil 3: Figure S3. Immunocytostaining of the HIF-1a localization in COL-XFM and XFM cells cultured in the presence of YC-1 under cobalt chloride-induced hypoxia conditions. Single cell analysis. Single channels (HIF-1a, DAPI, and vimentin) are shown in gray. The fluorescence intensities of HIF-1a (green) and nucleus (DAPI, magenta) are depicted by the dashed line (yellow) in a single cell, cell morphology was determined by vimentin (red), as shown in the merged images. Scale bars, $50 \mu \mathrm{m}$.

Additional file 4: Figure S4. Presence of collagen types IV (COL4), VII (COL7), and fibronectin (FN) in COL-XFM and XFM cultures on day 20 post-seeding (overconfluent/multilayered state) was determined by immunohistofluorescence. The single channels (COL4, COL7, FN, and DAPI) are shown in gray and all nuclei in the merged channel are shown in magenta (DAPI). Scale bars, $100 \mu \mathrm{m}$.

Additional file 5: Figure S5. Cell size and cell internal complexity/ granularity were determined by flow cytometry analysis using forward scatter (FSC-A) and side scatter (SSC-A) parameters, respectively, in both COL-XFM and XFM multilayers on day 20 post-seeding.

Additional file 6: Figure S6. Flow cytometry of the reference samples. (A): Cells were dissociated from the confluent COL-XFM cultures on day 10 and the suspension of live cells were gated in the forward scatter (FSC-A) and side scatter (SSC-A) dot plots to eliminate cell debris and aggregates. (B): For JC-1 staining, unstained cells were used as a negative control to determine background levels and autofluorescence. Cells cultured for 7 days without media change were used as a positive control: damaged mitochondria were confirmed by decrease of JC-1 fluorescence aggregates (and increase of JC-1 monomers).

\section{Abbreviations}

DPSCs: Dental pulp stem cells; MSCs: Mesenchymal stem cells; FBS: Fetal bovine serum; XFM: Xenogeneic serum-free culture medium; EDTA: Ethylenediaminetetraacetic acid; TEM: Transmission electron microscope; SD: Standard deviation; PFA: Paraformaldehyde; RT: Room temperature; HE: Hematoxylin and eosin; HIF-1a: Hypoxia-inducible factor-1 alpha; Ig: Immunoglobulin; DAPI: 4',6-Diamidino-2-phenylindole; RTPCR: Reverse transcription-polymerase chain reaction; $\mathrm{CoCl}_{2}$ : Cobalt chloride; COL1: Collagen type I; PB: Phosphate buffer solution; TUNEL: Terminal deoxynucleotidyl transferase-mediated deoxyuridine triphosphate nick end labeling; COL: Type I collagen; COL-XFM cells: DPSCs cultured on COL in XFM; XFM cells: DPSCs cultured on fibronectin in XFM; ECM: Extracellular matrix

\section{Acknowledgements}

The authors thank Yuji Watanabe (The University of Tokyo) for providing technical assistance.

\section{Authors' contributions}

MM contributed to conception and design, financial support, collection and assembly of data, data analysis and interpretation, and drafting the manuscript. HS contributed to collection and assembly of data, and data analysis and interpretation. TN contributed to conception and design, financial support, data analysis and interpretation, drafting and editing the manuscript, and final approval of the manuscript. All authors read and approved the final manuscript.

\section{Funding}

This work was supported by a Grant-in-Aid for Research Activity Start-up (Nos. JP19K21403 and JP18H06317 to M.M.), a Grant-in-Aid for Scientific Research (B) (No. 15 H05046 to T.N.), and a Grant-in-Aid for Young Scientists (A) (No. 24689073 to T.N.) from the Japan Society for the Promotion of Science (JSPS KAKENHI), and it was supported in part by a research grant (2017 to

T.N.) from the Nippon Dental University.

\section{Availability of data and materials}

The datasets supporting the conclusions of this article are included within the article and its additional files.

\section{Ethics approval and consent to participate}

The present study was approved by the ethics committee of Nippon Dental University School of Life Dentistry at Tokyo (No. NDU-T2013-10) and was conducted in accordance with the Declaration of Helsinki. Written informed consent was obtained from all individual donors after detailed explanation of the procedure and the intended use of the tissues.

\section{Consent for publication}

Not applicable.

\section{Competing interests}

The authors declare that they have no competing interests.

\section{Author details}

'Department of Life Science Dentistry, The Nippon Dental University, 1-9-20 Fujimi, Chiyoda-ku, Tokyo 102-8159, Japan. ${ }^{2}$ Department of Developmental and Regenerative Dentistry, The Nippon Dental University School of Life Dentistry at Tokyo, 1-9-20 Fujimi, Chiyoda-ku, Tokyo 102-8159, Japan. ${ }^{3}$ Medical Proteomics Laboratory, The Institute of Medical Science, The University of Tokyo, 4-6-1 Shirokanedai, Minato-ku, Tokyo 108-8639, Japan.

Received: 8 May 2020 Revised: 26 May 2020

Accepted: 12 June 2020 Published online: 14 July 2020

\section{References}

1. Kobayashi T, Torii D, Iwata T, Izumi Y, Nasu M, Tsutsui TW. Characterization of proliferation, differentiation potential, and gene expression among clonal cultures of human dental pulp cells. Hum Cell. 2020.

2. Kong Y, Hu X, Zhong Y, Xu K, Wu B, Zheng J. Magnesium-enriched microenvironment promotes odontogenic differentiation in human dental pulp stem cells by activating ERK/BMP2/Smads signaling. Stem Cell Res Ther. 2019;10:378. 
3. Fujii Y, Kawase-Koga Y, Hojo H, Yano F, Sato M, Chung UI, et al. Bone regeneration by human dental pulp stem cells using a helioxanthin derivative and cell-sheet technology. Stem Cell Res Ther. 2018;9:24.

4. Hu J, Cao Y, Xie Y, Wang H, Fan Z, Wang J, et al. Periodontal regeneration in swine after cell injection and cell sheet transplantation of human dental pulp stem cells following good manufacturing practice. Stem Cell Res Ther. 2016;7:130.

5. Mehrotra P, Tseropoulos G, Bronner ME, Andreadis ST. Adult tissue-derived neural crest-like stem cells: sources, regulatory networks, and translational potential. Stem Cells Transl Med. 2020;9:328-41.

6. Zhang C, Zhang Y, Feng Z, Zhang F, Liu Z, Sun X, et al. Therapeutic effect of dental pulp stem cell transplantation on a rat model of radioactivityinduced esophageal injury. Cell Death Dis. 2018;9:738.

7. Omi M, Hata M, Nakamura N, Miyabe M, Ozawa S, Nukada H, et al. Transplantation of dental pulp stem cells improves long-term diabetic polyneuropathy together with improvement of nerve morphometrical evaluation. Stem Cell Res Ther. 2017:8:279.

8. Mead B, Logan A, Berry M, Leadbeater W, Scheven BA. Dental pulp stem cells: a novel cell therapy for retinal and central nervous system repair. Stem Cells. 2017;35:61-7.

9. Martínez-Sarrà E, Montori S, Gil-Recio C, Núñez-Toldrà R, Costamagna D, Rotini A, et al. Human dental pulp pluripotent-like stem cells promote wound healing and muscle regeneration. Stem Cell Res Ther. 2017;8:175.

10. Ishkitiev N, Yaegaki K, Imai T, Tanaka T, Fushimi N, Mitev V, et al. Novel management of acute or secondary biliary liver conditions using hepatically differentiated human dental pulp cells. Tissue Eng Part A. 2015;21:586-93.

11. Gronthos S, Mankani M, Brahim J, Robey PG, Shi S. Postnatal human dental pulp stem cells (DPSCs) in vitro and in vivo. Proc Natl Acad Sci U S A. 2000; 97:13625-30.

12. Huang GT, Gronthos S, Shi S. Mesenchymal stem cells derived from dental tissues vs. those from other sources: their biology and role in regenerative medicine. J Dent Res. 2009;88:792-806.

13. Thompson M, Mei SHJ, Wolfe D, Champagne J, Fergusson D, Stewart DJ, et al. Cell therapy with intravascular administration of mesenchymal stromal cells continues to appear safe: an updated systematic review and metaanalysis. EClinicalMedicine. 2020;19:100249.

14. Kabat M, Bobkov I, Kumar S, Grumet M. Trends in mesenchymal stem cell clinical trials 2004-2018: is efficacy optimal in a narrow dose range? Stem Cells Transl Med. 2020;9:17-27.

15. Andrukhov O, Behm C, Blufstein A, Rausch-Fan X. Immunomodulatory properties of dental tissue-derived mesenchymal stem cells: implication in disease and tissue regeneration. World J Stem Cells. 2019;11:604-17.

16. Kusuma GD, Carthew J, Lim R, Frith JE. Effect of the microenvironment on mesenchymal stem cell paracrine signaling: opportunities to engineer the therapeutic effect. Stem Cells Dev. 2017:26:617-31.

17. De Luca M, Aiuti A, Cossu G, Parmar M, Pellegrini G, Robey PG. Advances in stem cell research and therapeutic development. Nat Cell Biol. 2019;21:801-11.

18. Sipp D, Robey PG, Turner L. Clear up this stem-cell mess. Nature. 2018;561: 455-7.

19. Shi X, Mao J, Liu Y. Pulp stem cells derived from human permanent and deciduous teeth: biological characteristics and therapeutic applications. Stem Cells Transl Med. 2020;9:445-64.

20. Janowicz K, Mozdziak P, Bryja A, Kempisty B, Dyszkiewicz-Konwińska M. Human dental pulp stem cells: recent findings and current research. Med J Cell Biol. 2019;7:119-24.

21. Ohkoshi S, Hirono H, Nakahara T, Ishikawa H. Dental pulp cell bank as a possible future source of individual hepatocytes. World J Hepatol. 2018;10: 702-7.

22. Liu J, Yu F, Sun Y, Jiang B, Zhang W, Yang J, et al. Concise reviews: characteristics and potential applications of human dental tissue-derived mesenchymal stem cells. Stem Cells. 2015;33:627-38

23. Odontology prize 2017. Odontology. 2017;105:391.

24. Tamaki $Y$, Nakahara T, Ishikawa H, Sato $S$. In vitro analysis of mesenchymal stem cells derived from human teeth and bone marrow. Odontology. 2013; 101:121-32

25. Sundin M, Ringdén O, Sundberg B, Nava S, Götherström C, Le Blanc K. No alloantibodies against mesenchymal stromal cells, but presence of anti-fetal calf serum antibodies, after transplantation in allogeneic hematopoietic stem cell recipients. Haematologica. 2007;92:1208-15.

26. Mackensen A, Dräger $\mathrm{R}$, Schlesier M, Mertelsmann $\mathrm{R}$, Lindemann A. Presence of IgE antibodies to bovine serum albumin in a patient developing anaphylaxis after vaccination with human peptide-pulsed dendritic cells. Cancer Immunol Immunother. 2000:49:152-6.

27. Palama MEF, Shaw GM, Carluccio S, Reverberi D, Sercia L, Persano L, et al. The secretome derived from mesenchymal stromal cells cultured in a xenofree medium promotes human cartilage recovery in vitro. Front Bioeng Biotechnol. 2020:8:90.

28. Bakopoulou A, Apatzidou D, Aggelidou E, Gousopoulou E, Leyhausen G, Volk J, et al. Isolation and prolonged expansion of oral mesenchymal stem cells under clinical-grade, GMP-compliant conditions differentially affects "stemness" properties. Stem Cell Res Ther. 2017:8:247.

29. Chase LG, Yang S, Zachar V, Yang Z, Lakshmipathy U, Bradford J, et al. Development and characterization of a clinically compliant xeno-free culture medium in good manufacturing practice for human multipotent mesenchymal stem cells. Stem Cells Transl Med. 2012;1:750-8.

30. Wang Y, Wu H, Yang Z, Chi Y, Meng L, Mao A, et al. Human mesenchymal stem cells possess different biological characteristics but do not change their therapeutic potential when cultured in serum free medium. Stem Cell Res Ther. 2014;5:132

31. Chen G, Yue A, Ruan Z, Yin Y, Wang R, Ren Y, et al. Human umbilical cordderived mesenchymal stem cells do not undergo malignant transformation during long-term culturing in serum-free medium. PLoS One. 2014;9:e98565.

32. Sato K, Itoh T, Kato T, Kitamura Y, Kaul SC, Wadhwa R, et al. Serum-free isolation and culture system to enhance the proliferation and bone regeneration of adipose tissue-derived mesenchymal stem cells. In Vitro Cell Dev Biol Anim. 2015:51:515-29.

33. Murabayashi D, Mochizuki M, Tamaki Y, Nakahara T. Practical methods for handling human periodontal ligament stem cells in serum-free and serumcontaining culture conditions under hypoxia: implications for regenerative medicine. Hum Cell. 2017;30:169-80.

34. Trubiani O, Piattelli A, Gatta V, Marchisio M, Diomede F, D'Aurora M, et al. Assessment of an efficient xeno-free culture system of human periodontal ligament stem cells. Tissue Eng Part C Methods. 2015;21:52-64.

35. Qu C, Brohlin M, Kingham PJ, Kelk P. Evaluation of growth, stemness, and angiogenic properties of dental pulp stem cells cultured in cGMP xeno-/ serum-free medium. Cell Tissue Res. 2020;380:93-105.

36. Abdel Moniem EM, El-Batran MM, Halawa AM, Gomaa DH, Eldeen GN, Aly RM. Optimizing a serum-free/xeno-free culture medium for culturing and promoting the proliferation of human dental pulp stem cells. Stem Cell Investig. 2019;6:15

37. Mochizuki M, Nakahara T. Establishment of xenogeneic serum-free culture methods for handling human dental pulp stem cells using clinically oriented in-vitro and in-vivo conditions. Stem Cell Res Ther. 2018:9:25.

38. Ishkitiev N, Yaegaki K, Imai T, Tanaka T, Nakahara T, Ishikawa H, et al. Highpurity hepatic lineage differentiated from dental pulp stem cells in serumfree medium. J Endod. 2012;38:475-80.

39. Coates DE, Alansary M, Friedlander L, Zanicotti DG, Duncan WJ. Dental pulp stem cells in serum-free medium for regenerative medicine. J Royal Soc New Zealand. 2020;50:80-90

40. Van Pham P, Nguyen HT, Vu NB. Evolution of stem cell products in medicine: future of off-the-shelf products. In: Pham PV, editor. Stem cell drugs - a new generation of biopharmaceuticals. Cham: Springer International Publishing; 2018. p. 93-118.

41. Tominaga N, Nakahara T, Nasu M, Satoh T. Isolation and characterization of epithelial and myogenic cells by "fishing" for the morphologically distinct cell types in rat primary periodontal ligament cultures. Differentiation. 2013;85:91-100.

42. Shinagawa-Ohama R, Mochizuki M, Tamaki Y, Suda N, Nakahara T. Heterogeneous human periodontal ligament-committed progenitor and stem cell populations exhibit a unique cementogenic property under in vitro and in vivo conditions. Stem Cells Dev. 2017;26:632-45.

43. Xiao L, Tsutsui T. Characterization of human dental pulp cells-derived spheroids in serum-free medium: stem cells in the core. J Cell Biochem. 2013:114:2624-36.

44. Nakahara T, Tominaga N, Toyomura J, Tachibana T, Ide $Y$, Ishikawa H. Isolation and characterization of embryonic ameloblast lineage cells derived from tooth buds of fetal miniature swine. In Vitro Cell Dev Biol Anim. 2016; 52:445-53.

45. Wang L, Wu B, Zhang Y, Tian Z. Hypoxia promotes the proliferation of MC3T3-E1 cells via the hypoxia-inducible factor-1a signaling pathway. Mol Med Rep. 2015;12:5267-73.

46. Park IH, Kim KH, Choi HK, Shim JS, Whang SY, Hahn SJ, et al. Constitutive stabilization of hypoxia-inducible factor alpha selectively promotes the self- 
renewal of mesenchymal progenitors and maintains mesenchymal stromal cells in an undifferentiated state. Exp Mol Med. 2013:45:e44.

47. Popov C, Radic T, Haasters F, Prall WC, Aszodi A, Gullberg D, et al. Integrins a2 $\beta 1$ and $\alpha 11 \beta 1$ regulate the survival of mesenchymal stem cells on collagen I. Cell Death Dis. 2011;2:e186.

48. Murphy KC, Hoch Al, Harvestine JN, Zhou D, Leach JK. Mesenchymal stem cell spheroids retain osteogenic phenotype through a2 $\beta 1$ signaling. Stem Cells Transl Med. 2016;5:1229-37.

49. Larjava H, Koivisto L, Heino J, Häkkinen L. Integrins in periodontal disease. Exp Cell Res. 2014;325:104-10.

50. Marastoni S, Ligresti G, Lorenzon E, Colombatti A, Mongiat M. Extracellular matrix: a matter of life and death. Connect Tissue Res. 2008;49:203-6.

51. Barczyk M, Carracedo S, Gullberg D. Integrins. Cell Tissue Res. 2010;339:269-80.

52. Zeltz C, Gullberg D. The integrin-collagen connection--a glue for tissue repair? J Cell Sci. 2016:129:653-64.

53. Chen C, Tang $Q$, Zhang Y, Dai M, Jiang Y, Wang H, et al. Metabolic reprogramming by HIF-1 activation enhances survivability of human adipose-derived stem cells in ischaemic microenvironments. Cell Prolif. 2017:50:e12363.

54. Deschene K, Céleste C, Boerboom D, Theoret CL. Hypoxia regulates the expression of extracellular matrix associated proteins in equine dermal fibroblasts via HIF1. J Dermatol Sci. 2012;65:12-8.

55. Somaiah C, Kumar A, Mawrie D, Sharma A, Patil SD, Bhattacharyya J, et al. Collagen promotes higher adhesion, survival and proliferation of mesenchymal stem cells. PLoS One. 2015;10:e145068.

56. Rakian R, Block TJ, Johnson SM, Marinkovic M, Wu J, Dai Q, et al. Native extracellular matrix preserves mesenchymal stem cell "stemness" and differentiation potential under serum-free culture conditions. Stem Cell Res Ther. 2015;6:235

57. Chen XD, Dusevich V, Feng JQ, Manolagas SC, Jilka RL. Extracellular matrix made by bone marrow cells facilitates expansion of marrow-derived mesenchymal progenitor cells and prevents their differentiation into osteoblasts. J Bone Miner Res. 2007:22:1943-56.

\section{Publisher's Note}

Springer Nature remains neutral with regard to jurisdictional claims in published maps and institutional affiliations.

Ready to submit your research? Choose BMC and benefit from:

- fast, convenient online submission

- thorough peer review by experienced researchers in your field

- rapid publication on acceptance

- support for research data, including large and complex data types

- gold Open Access which fosters wider collaboration and increased citations

- maximum visibility for your research: over $100 \mathrm{M}$ website views per year

At $\mathrm{BMC}$, research is always in progress.

Learn more biomedcentral.com/submissions 